\title{
Phylogenetic patterns and phenotypic profiles of the species of plants and mammals farmed for food
}

\author{
Rubén Milla ${ }^{1 \star}$, Jesús M. Bastida1, Martin M. Turcotte ${ }^{2}$, Glynis Jones ${ }^{3}$, Cyrille Violle ${ }^{4}$, Colin P. Osborne ${ }^{5}$, \\ Julia Chacón-Labella ${ }^{1,19}$, ÊnioE. Sosinski Jr ${ }^{6}$, Jens Kattge ${ }^{7,8}$, Daniel C. Laughlin ${ }^{9}$, Estelle Forey ${ }^{10}$, \\ Vanessa Minden ${ }^{11,12}$, Johannes H. C. Cornelissen ${ }^{13}$, Bernard Amiaud ${ }^{14,20}$, Koen Kramer (15), \\ Gerhard Boenisch7, Tianhua He ${ }^{16}$, Valério D. Pillar ${ }^{10}{ }^{17}$ and ChaehoByun ${ }^{18}$
}

The origins of agriculture were key events in human history, during which people came to depend for their food on small numbers of animal and plant species. However, the biological traits determining which species were domesticated for food provision, and which were not, are unclear. Here, we investigate the phylogenetic distribution of livestock and crops, and compare their phenotypic traits with those of wild species. Our results indicate that phylogenetic clustering is modest for crop species but more intense for livestock. Domesticated species explore a reduced portion of the phenotypic space occupied by their wild counterparts and have particular traits in common. For example, herbaceous crops are globally characterized by traits including high leaf nitrogen concentration and tall canopies, which make them fast-growing species and proficient competitors. Livestock species are relatively large mammals with low basal metabolic rates, which indicate moderate to slow life histories. Our study therefore reveals ecological differences in domestication potential between plants and mammals. Domesticated plants belong to clades with traits that are advantageous in intensively managed high-resource habitats, whereas domesticated mammals are from clades adapted to moderately productive environments. Combining comparative phylogenetic methods with ecologically relevant traits has proven useful to unravel the causes and consequences of domestication.

T he plant and animal species domesticated for human food supply represent only a small fraction of global biodiversity. Of around 370,000 extant flowering plants ${ }^{1}$, only 1,000-2,000 have undergone some form of domestication for that purpose ${ }^{2-4}$. Similarly, humans have domesticated 20-31 species of mammals for food ${ }^{5,6}$, from $\sim 5,400$ species contemporary to late Palaeolithic people 7 . The taxonomic distribution of species used for farming seems non-random ${ }^{5,8}$, such that certain families include numerous domesticated species (for example, grasses and legumes among flowering plants, and bovids and camelids among mammals), while many others contain none. An uneven phylogenetic distribution of the species that became domesticated would imply that certain combinations of phenotypic traits are more adaptive for husbandry, if these traits are phylogenetically conserved ${ }^{9}$. However, global comparative analyses between domesticates and wild relatives are rare $^{10-12}$ or consider taxonomically and/or geographically restricted groups of species ${ }^{13,14}$. Filling that gap would direct agricultural sciences towards the phylogenetic groups and traits that could be pursued for new food sources. In addition, investigating such patterns at a global scale, while explicitly linking phylogenetic and trait distributions, would highlight the usefulness of the tools and concepts of evolutionary ecology to address questions at its interface with agricultural sciences and archaeology.

The phenotypes of current livestock and crops are the result of early domestication processes and millennia of unconscious and deliberate selection under farming ${ }^{15}$. Evolution under farming has caused the traits of domesticated species to change under shifting selective forces ${ }^{13}$. For example, local breeding preserves DNA mutations that would otherwise be eliminated by natural selection and thereby offset the sampling effects of early farming ${ }^{4}$. In fact, diversifying selection has promoted remarkable variance in the size of crop seeds or in animal coat colours ${ }^{5,16}$. Conversely, directional selection for productivity has resulted in the convergence of a number of livestock and crop traits, that is, the domestication

'Departamento de Biologia, Geología, Física y Química Inorgánica, Universidad Rey Juan Carlos, Móstoles, Spain. ²Department of Biological Sciences, University of Pittsburgh, Pittsburgh, PA, USA. ${ }^{3}$ Department of Archaeology, University of Sheffield, Sheffield, UK. ${ }^{4}$ CEFE UMR 5175, CNRS - Université de Montpellier - Université Paul-Valéry Montpellier - EPHE, Montpellier, France. ${ }^{5}$ Department of Animal and Plant Sciences, University of Sheffield, Sheffield, UK. ${ }^{6}$ Embrapa Clima Temperado, Pelotas, Brazil. ${ }^{7}$ Max Planck Institute for Biogeochemistry, Jena, Germany. ${ }^{8}$ German Centre for Integrative Biodiversity Research (iDiv) Halle-Jena-Leipzig, Leipzig, Germany. ${ }^{9}$ Department of Botany, University of Wyoming, Laramie, WY, USA. ${ }^{10}$ Laboratoire Ecodiv URA/EA 1293, IRSTEA, FR CNRS 3730 SCALE, UFR Sciences et Techniques, Normandie Université, Université de Rouen, Mont Saint Aignan, France. "Department of Biology, Ecology and Biodiversity, Vrije Universiteit Brussel, Brussels, Belgium. ${ }^{12}$ Landscape Ecology Group, Institute of Biology and Environmental Sciences, Oldenburg, Germany. ${ }^{13}$ Systems Ecology, Department of Ecological Science, Faculty of Science, Vrije Universiteit Amsterdam, Amsterdam, The Netherlands. ${ }^{14}$ UMR Ecologie et Ecophysiologie Forestières, Faculté des Sciences et Technologies, Université de Lorraine, INRA, Vandoeuvre-lès-Nancy, France. ${ }^{15}$ Wageningen Environmental Research, Wageningen University, Wageningen, The Netherlands. ${ }^{16}$ School of Molecular and Life Sciences, Curtin University, Perth, Western Australia, Australia. ${ }^{17}$ Department of Ecology, Universidade Federal do Rio Grande do Sul, Porto Alegre, Brazil. ${ }^{18}$ School of Civil and Environmental Engineering, Yonsei University, Seoul, South Korea. ${ }^{19}$ Present address: Department of Environmental System Science, Swiss Federal Institute of Technology, ETH Zurich, Zurich, Switzerland, ${ }^{20}$ Deceased: Bernard Amiaud. *e-mail: ruben.milla@gmail.com 


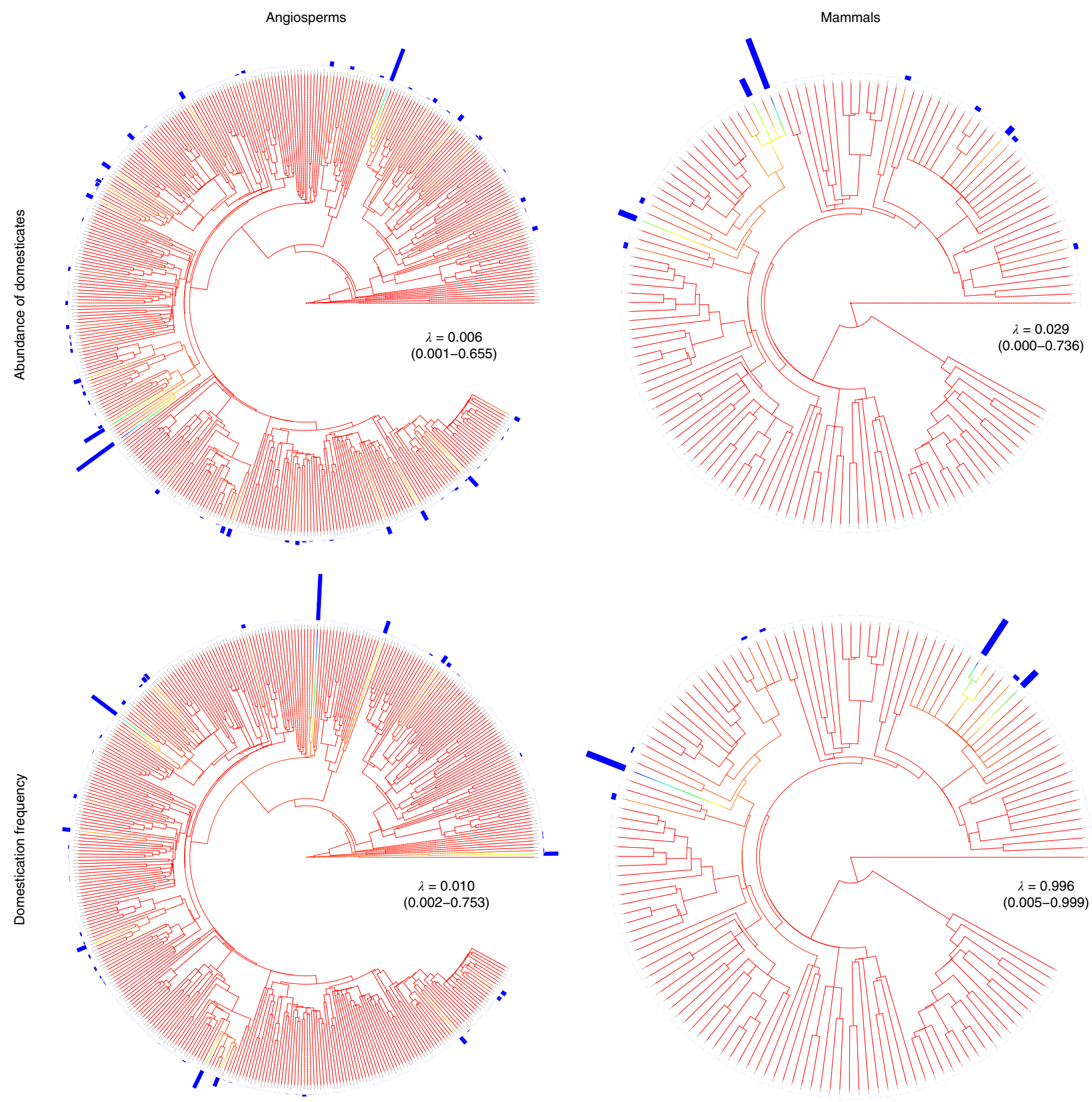

Fig. 1 | Distribution of the abundance of food domesticates and frequency of domestication events across mammalian and angiosperm families. Length of blue bars are relative abundance of domesticated species (proportion of all domesticated species that are found within a given family), and of domestication frequencies (proportion of all species in a family that were domesticated). Raw data can be found in Supplementary Table 1 and Supplementary Data 1. The figure can be magnified online to view family identities in the phylogeny. Colours of tree edges correspond to domestication abundances or frequencies, according to a gradient of increasing rates from zero (red) to one (blue). Within each phylogeny, the inset indicates the posterior mode of the phylogenetic signal $(\lambda)$, together with its $95 \%$ credible interval.

syndrome ${ }^{5,9,17}$ (but see refs ${ }^{18,19}$ ). Domestication syndrome traits include increased docility or reductions in brain size in livestock ${ }^{5,6,20}$ and increased palatability or the loss of seed dispersal mechanisms in crop plants ${ }^{4,9}$.

Adaptations to the farming environment might also promote convergent evolution via natural selection ${ }^{13}$. For instance, evolution in high-resource croplands should select for suites of traits that enable fast resource acquisition and rapid canopy closure, according to trait-based ecology ${ }^{21}$. A few recent studies have partially supported this view, showing that several crop plants display traits indicative of high competitive ability ${ }^{14,22-24}$. Therefore, if directional and stabilizing selection are strong, the phenotypic diversity of domesticated species will be low, adding to early domestication bottlenecks. In contrast, diversifying selection, associated with centuries-long 

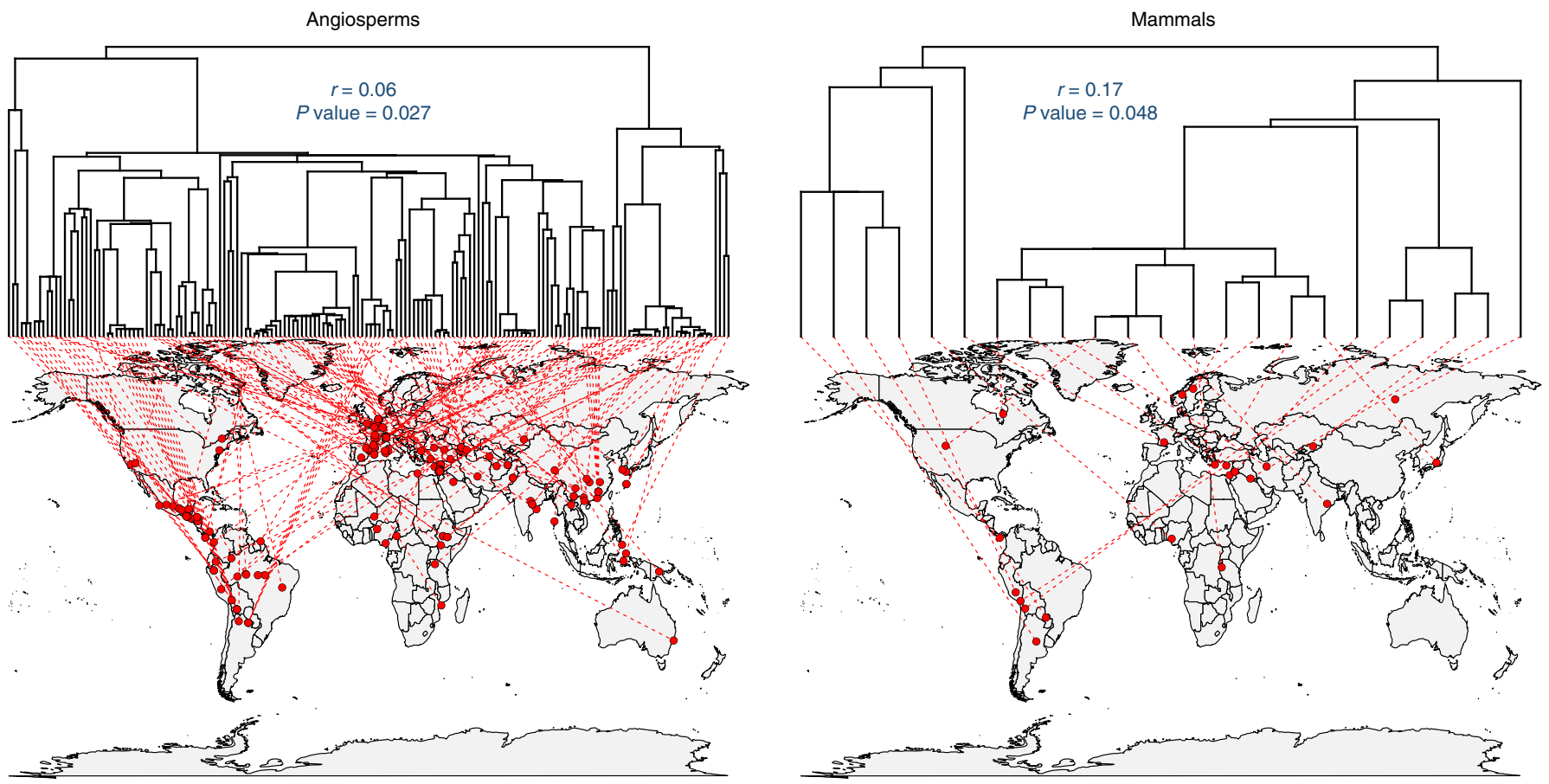

Fig. 2 | Phylogeographic distribution of the putative place of origin of food domesticates included in phenotypic space analyses. Locations in the map are medians of GBIF coordinate records for the putative wild progenitor of each domesticate. Data were retrievable for 168 wild progenitors of crops, out of 181 crop species, and for all of the 23 wild progenitors of mammal domesticates. Insets: Mantel test statistics for the correlation between phylogenetic and geographic distance matrices. See Supplementary Methods for further details.

geographic expansion under farming, is expected to promote the widening of phenotypic spaces ${ }^{16}$. The net effects of the early filtering of wild species, of subsequent domestication processes and of later crop and livestock evolution on the phenotypic spaces explored by domesticated species remain unknown. Here we show the results of broad-scale phylogenetic analyses addressing whether domesticates are a limited phylogenetic and phenotypic sample of wild plants and animals, and uncovering traits that distinguish domesticated species from wild species.

We used phylogenetic comparative methods to investigate the phylogenetic patterns of domestication events, and to determine whether domesticates are phenotypically distinct from their wild relatives. We did this by compiling and analysing two large datasets. First, we compiled a database on the distribution of species domesticated for food across mammal and angiosperm families and genera. With that dataset, we investigated evolutionary patterns of the relative abundance of domesticated species (proportion of all domesticated species within a given genus or family), and of domestication frequencies (proportion of all species in a genus or family that were domesticated) across mammal and angiosperm phylogenies. Second, we put together a large-scale database of three key phenotypic traits for farm mammals (size-corrected basal metabolic rate, adult body mass and neonate body mass) and crop plants (leaf nitrogen content, plant canopy height and seed dry mass) across a broad sample of domesticated (23 mammals, 181 angiosperms) and wild (885 mammals, 2,943 angiosperms) species. Traits were selected based on their key functional relevance for resource-useacquisition, life-history and ecological strategies, both for domesticated and for wild species ${ }^{21,25-28}$. Using this second dataset, we compared the phenotypic spaces of domesticates to those of their wild relatives. We set out to address three questions: (1) How are food domesticates distributed across the phylogenies of mammals and angiosperms? (2) Do livestock and crop species have particular phenotypic profiles, when compared with their wild counterparts? And, if so, (3) do the phenotypic traits of domesticated species fall within the trait space exhibited by wild species, or do they extend their phenotypic space beyond the boundaries set by wild plants and animals?

\section{Results}

Some families and genera contain more livestock or crop species than others. Livestock species were found in only ten families. In particular, Bovidae harbour $\sim 40 \%$ of domesticated species (Supplementary Table 1), and only 22 genera of mammals contain domesticated species (Supplementary Table 1). In contrast, crop species were distributed across 120 families and 453 genera of angiosperms (Supplementary Data 1). Fabaceae, Solanum and Poaceae are examples of taxa yielding high proportions of crops. The abundances of domesticated species were far from randomly distributed across families and growth forms (plants) or dietary types (mammals), both for mammals and angiosperms (Supplementary Table 2). We next investigated whether the above deviations from a random distribution were phylogenetically structured.

In general, we found that the phylogenetic structure of domestication abundances and frequencies was modest for plants, though stronger for mammals. First, local indicators of phylogenetic affinity (LIPAs) indicated that ca. $90 \%$ of the plant families hosting domesticated species were randomly distributed with respect to the domestication status of their phylogenetic neighbourhood (Supplementary Data 2). Approximately $10 \%$ of angiosperm families departed from a non-significant LIPA score (Supplementary Data 2). However, such departure signalled over-dispersion (that is, focal family surrounded by families without domesticated species more than the random expectation), rather than clustering (Supplementary Data 2). Interestingly, ca. 10\% of angiosperm families include Fabaceae, Poaceae, Rosaceae, Solanaceae or Asteraceae, which host crops of high agricultural relevance. For mammals, four (Suidae, Cervidae, Caviidae, and Cunilidae) out of ten families with domesticated species had at least one LIPA score indicating phylogenetic clustering, either for abundances or frequencies. Second, we 

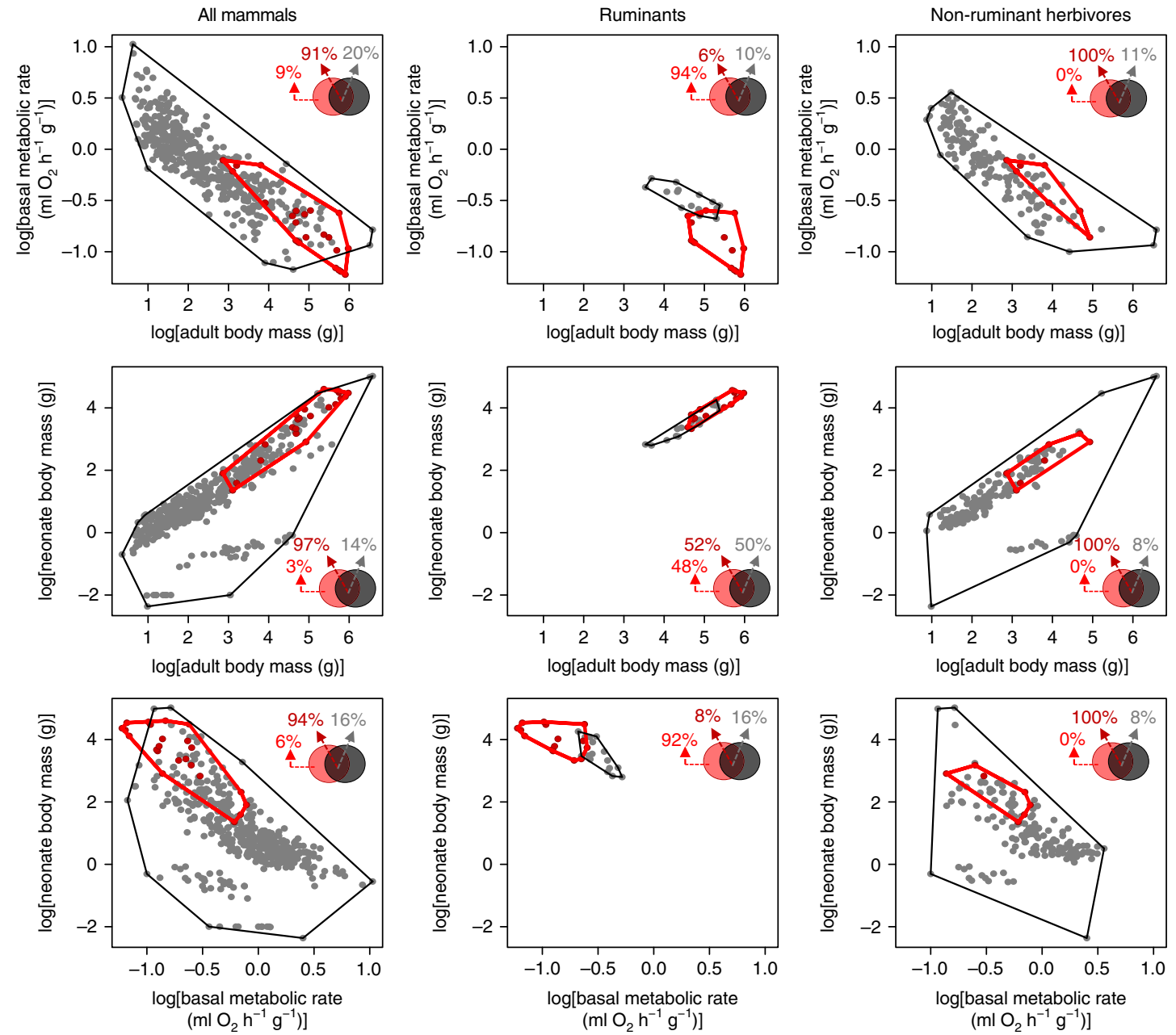

Fig. 3 | Phenospace occupancy of livestock and wild mammals. Separate plots are shown for all mammals, ruminants and non-ruminant herbivores. Grey dots and red dots are wild and domesticated mammals, respectively. Black and red polygons are convex hulls for wild and domesticated mammals, respectively. Numbers in the insets are percentage of convex-hull area of domesticates outside the wild boundary (light red), of domesticates inside the wild boundary (dark red-grey) and of wild space occupied by domesticates (grey).

investigated phylogenetic clustering at the scale of the whole phylogenetic tree. When two contrasting evolutionary models were compared (that is a Brownian-motion model of evolution, representing strong phylogenetic structure, versus a star phylogeny, representing full phylogenetic independence), phylogenetically independent models showed better fit to the data than Brownian-motion models, both for mammals and angiosperms (Supplementary Tables 3 and 4). Finally, global phylogenetic clustering was investigated with a gradual approach (phylogenetic signal), which complemented the binary (non-phylo versus phylo) comparison of phylogenetic models above. The frequency of domestication events generally showed a phylogenetic signal (Fig. 1 and Supplementary Fig. 1, but see angiosperm families in Fig. 1). Domestication abundance, instead, showed low or no phylogenetic signal in angiosperms, low signal in mammals at the family scale and high signal when mammals were examined separately for each dietary type (Fig. 1 and Supplementary Fig. 1).

The subset of domesticated species used for phenotypic space analyses covered a wide range of phylogenetic and geographic origins (Fig. 2). In spite of this taxonomic and geographic diversity, domesticated species were distributed across a portion of the phenotypic space generally occupied by wild species, and rarely fell beyond the bounds set by wild mammals and plants (Figs. 3 and 5 , and Supplementary Tables 5 and 6, but see ruminant livestock in
Fig. 3). Livestock occupied a small subset of the phenotypic spaces of wild mammals (Fig. 3 and Supplementary Table 5). Within the common phenotypic boundary occupied by wild and domesticated mammals, livestock species were, on average, larger as adults and gave birth to larger neonates, but had lower basal metabolic rates, compared with their wild counterparts (Fig. 4 and Supplementary Tables 7 and 9). Those phenotypic biases were upheld when investigated separately for ruminants and non-ruminants, though domestic ruminants lied mostly outside the phenotypic boundaries of wild ruminants. In contrast, domesticated crops have been selected from a wide range of botanical diversity in the three focal traits (Fig. 5 and Supplementary Table 6). An exception to that pattern is the small phenotypic space occupied by domesticated graminoids (grass-like monocot plants), compared with their wild analogues (Fig. 5 and Supplementary Table 6). However, although crops were phenotypically diverse, they occupied some regions of the phenotypic space more than others, which lead to phenotypic differentiation when compared with wild plants. Specifically, herbaceous crops, both graminoid and non-graminoid, were generally larger plants with larger seeds, and with leaves with higher nitrogen content, than their wild counterparts (Fig. 6 and Supplementary Tables 8 and 10). Woody crops were more similar to wild woody plants, though they consistently had larger seeds (Fig. 6 and Supplementary Tables 8 and 10). The phenotypic departure of domesticated species from 

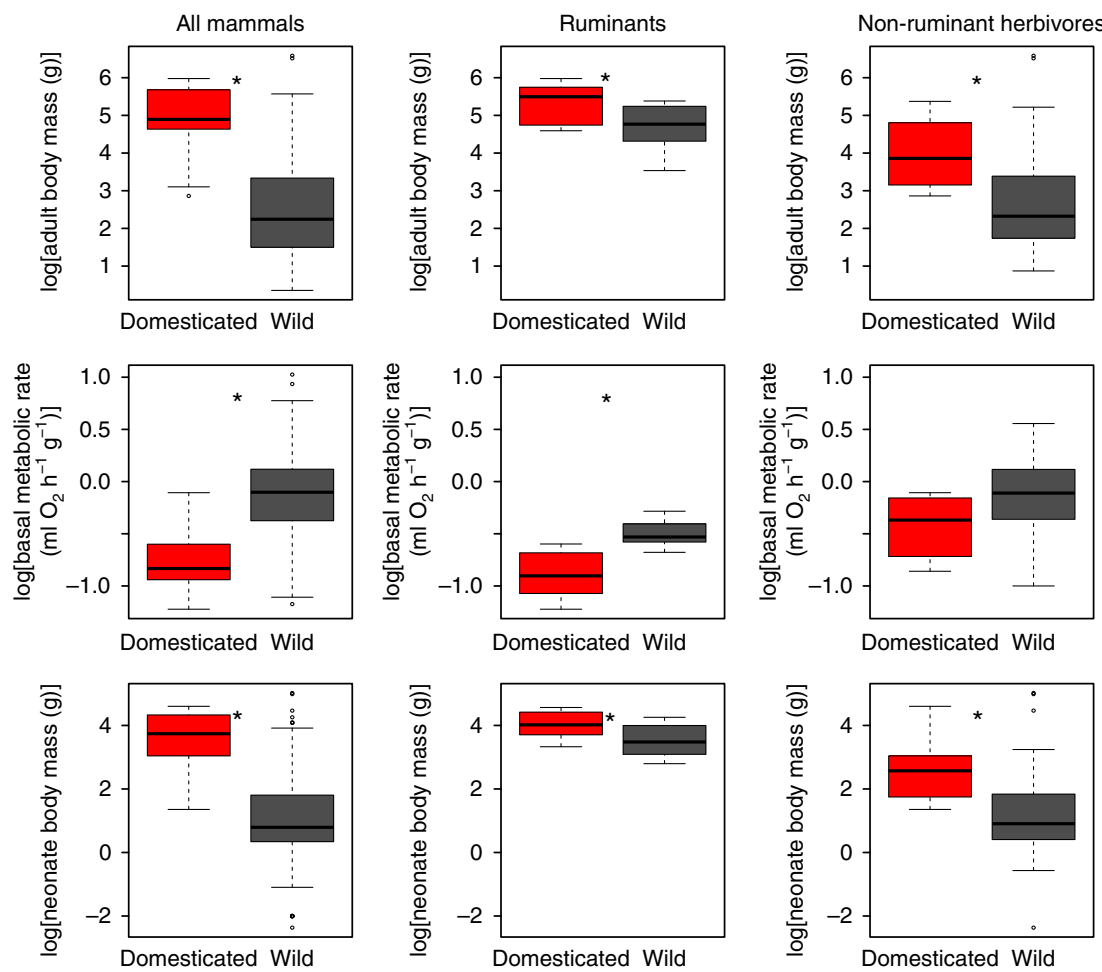

Fig. 4 | Phenotypic differentiation between livestock and wild mammals. Separate panels are shown for all mammals, non-ruminants and ruminants. Asterisks indicate statistically significant differences at $P \leq 0.05$ between domestication statuses according to phylogenetic ANOVAs (Supplementary Table 7). Centre line, median; box limits, upper and lower quartiles; whiskers, lowest/highest data point still within $1.5 \times$ interquartile range; points, data exceeding whisker bounds.

the trait medians of their wild counterparts was generally unrelated to the differences in geographic origin, climate at geographic origin, or antiquity of domestication of crop and livestock species (Supplementary Table 11).

\section{Discussion}

Our results provide a quantitative global test of the long-standing hypothesis that domestication events have a strong phylogenetic structure. We found only weak evidence for phylogenetic clustering in crops, but stronger evidence of such clustering in livestock species. Interestingly, the non-random phylogenetic distribution of species that became domesticated was associated with non-random phenotypic spaces of crops and farm mammals. Livestock species had moderate to low basal metabolic rates, gave birth to large offspring and were large adults. Herbaceous crops had high leaf nitrogen content (an indicator of photosynthetic rates), were large as adults and produced large seeds. These results show that domesticated mammals and plants occupy specific portions of the spectra of phenotypic variation ${ }^{21,25-28}$. Despite such phenotypic differentiation, and in spite of substantial trait evolution during domestication ${ }^{16}$, domesticated species were rarely positioned outside the bounds of the phenotypic spaces set by the wild species of their kind, excluding ruminant livestock. These findings have important implications for understanding the potential of wild species for farming, the patterns of phenotypic convergence under domestication, and the adaptation of wild species to the environmental conditions of farming habitats ${ }^{13,18,20,29,30}$. In addition, we demonstrate that a macroevolutionary approach, scarcely embraced in the domestication literature, has strong potential to advance this field.

Our analyses showed that domesticated mammals represent a small subsample of the total phenotypic variation displayed by wild animals. This was expected, as livestock species are a very small fraction of all contemporary mammals. More unexpectedly, farmed mammals had middle to low basal metabolic rates and were middle to large adults and neonates. These results portray domesticated mammals as moderate to slow life-history strategists, that is, species with intermediate body temperatures, with moderately long juvenile periods, giving birth to few but relatively large offspring, and living for reasonably long timespans, according to the fastslow life-history framework ${ }^{26,27}$. Low basal metabolic rates, which might entail slow relative growth rates $^{31}$, are adaptive in unproductive and unpredictable environments in ruminants ${ }^{32}$, rodents ${ }^{33}$ and mammals in general ${ }^{27,28}$. In addition, a moderate-slow lifestyle might genetically associate with behavioural traits that are critical to animal domestication, such as boldness, tameness or sociality ${ }^{33-35}$, which remains to be investigated in more depth. Interestingly, many domesticated mammals evolved body size reduction after domestication $^{29,36}$. This suggests that the moderate-slow lifestyle of livestock identified in our current work is largely the result of early selection of wild animals, rather than of further evolution under farming. In livestock species that show such body size reductions during domestication, decreases in sexual dimorphism were also reported, which, following Rensch's rule ${ }^{37}$, might account for their overall smaller adult size ${ }^{5,29,38}$. Further studies advancing this line of enquiry should consider the wild progenitors of livestock species, account for sexual dimorphism, and address species domesticated for purposes beyond food provision, which clearly display body size reduction after domestication (for example, dogs or cats).

For crops, our results for non-woody plants are compatible with hypotheses positing that early human selection favoured traits advantageous in the fertile, disturbed habitats surrounding human settlements and early agricultural fields $s^{5,9,30}$. Herbaceous crops occupied only a portion of the phenotypic space of their wild counterparts, suggesting habitat filtering ${ }^{39}$. This is in line with previous case 

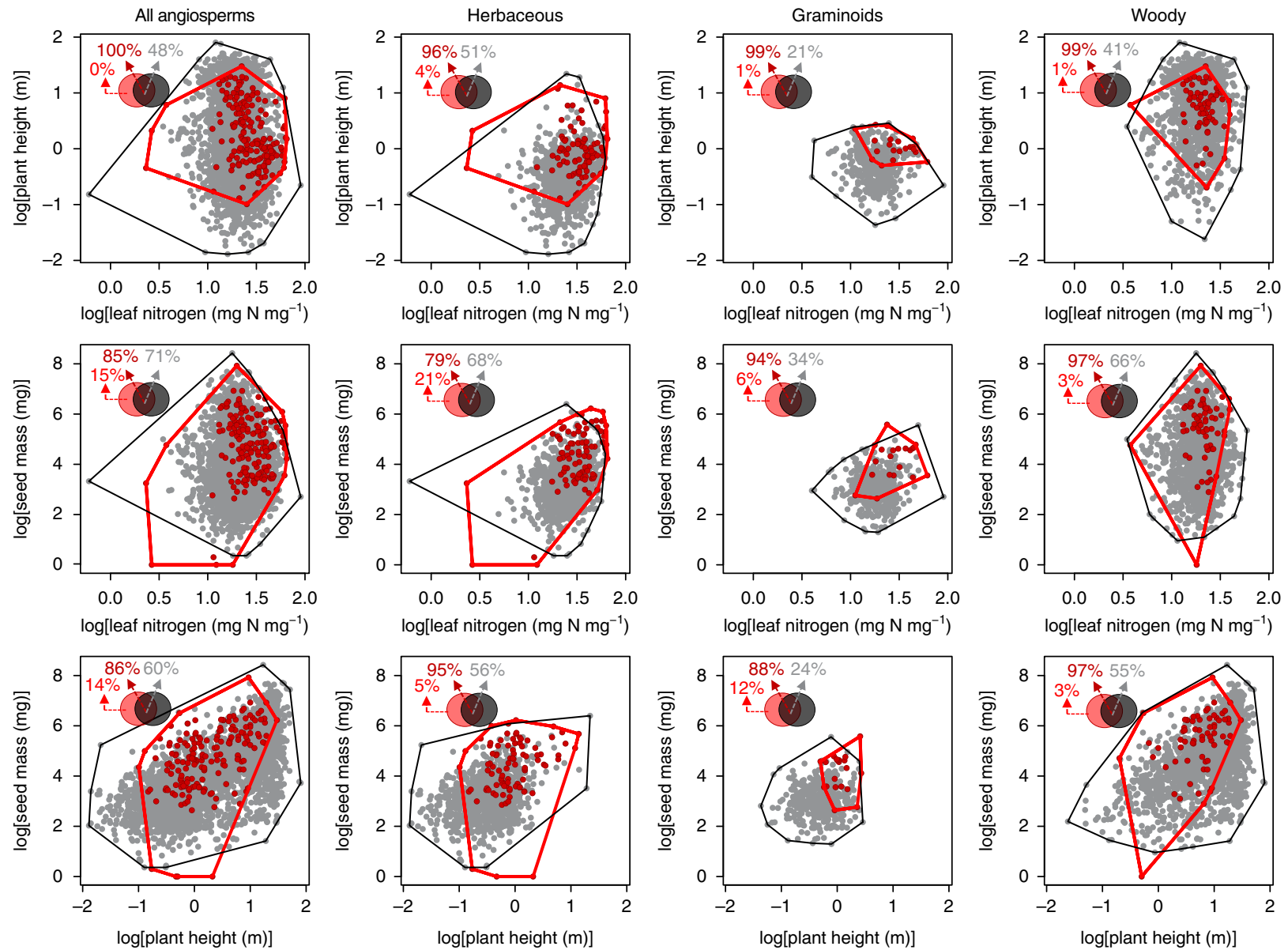

Fig. 5 | Phenospace occupancy of crops and wild angiosperms. Separate plots are shown for all angiosperms and for each growth form (that is herbaceous, graminoids and woody). Grey dots and red dots are wild and domesticated angiosperms, respectively. Black and red polygons are convex hulls for wild and domesticated angiosperms, respectively. Numbers in the insets are percentage of convex-hull area of domesticates outside the wild boundary (light red), of domesticates inside the wild boundary (dark red-grey) and of wild space occupied by domesticates (grey).

studies reporting that crops are a subsample of the phenotypic variation found in nature, and have not surpassed the biological limits observed for wild plants ${ }^{13,23,40,41}$, which suggests limitations of artificial selection to move phenotypes beyond what is observed in the wild. In addition, the phenotypic profiles described here indicate that herbaceous crops are fast-growing species (high leaf nitrogen content) and proficient competitors in resource-abundant environments (tall plants and large seeds) ${ }^{21,25}$, which would suit the ecological requirements of early agricultural habitats ${ }^{42}$. Such phenotypic differentiation passed unnoticed in the previous literature addressing smaller sets of crops, where crop-specific contrasting results were commonly reported ${ }^{14,22-24,41}$. This highlights the relevance of a global approach and the usage of the comparative method in this field. Woody crops yielded large seeds but, in contrast to herbs, were neither tall species nor species with high nitrogen content in their leaves. Multiple explanations might account for such discrepancy between growth forms, including trade-offs in resource allocation to fruit tissue, to vegetative growth, and to the maintenance of woody tissues, the clonal mode of propagation common to woody crops, or crop uses ${ }^{4,9,18,43}$.

A direct comparison of the phenotypic spaces of plants and mammals yielded an additional relevant insight. In plants, metabolism and size are largely decoupled ${ }^{25}$. In contrast, the evolution of metabolic rates and body size are coordinated in mammals ${ }^{44}$. Our trait analyses were consistent with these patterns, both for wild and domesticated species (Figs. 3 and 5). The phenotypes of domesticates therefore evolve under different constraints in plants and mammals. As a consequence, we found wider combinations of traits and larger phenotypic spaces for crops than for livestock. Endothermy, design of the vascular system and size-metabolism constraints might impede the simultaneous maximization of mass-specific growth rates and body size in mammals ${ }^{45}$, both of which are desirable traits for productive husbandry. Within these constraints, human selection for farming favoured animals with intermediate-high sizes, although at the cost of low metabolic rates, and thus probably modest relative growth rates. Breeding livestock that overcome size-metabolism constraints is therefore expected to be challenging. In contrast, crop plants occupied a wider part of the trade-off-free spectrum of metabolism (leaf nitrogen) versus size (plant and seed sizes), in line with their wild counterparts. Plant modularity and nitrogen transfer among modules, which underpin such uncoupling between metabolism and $\operatorname{size}^{46}$, might thus promote the greater phenotypic diversity of crops than livestock mammals, even when considered within plant growth forms. Breeding to simultaneously optimize variation in plant and organ sizes, and variation in metabolic rates, might be trade-off-free in plants.

Finally, we highlight two limitations of the current work. First, trait data come from plants and animals sampled in their typical habitats, which are different among species, and are undoubtedly so among organisms living in farm versus wild habitats. Thus, the phenotypic patterns encountered here came from a mixture of 

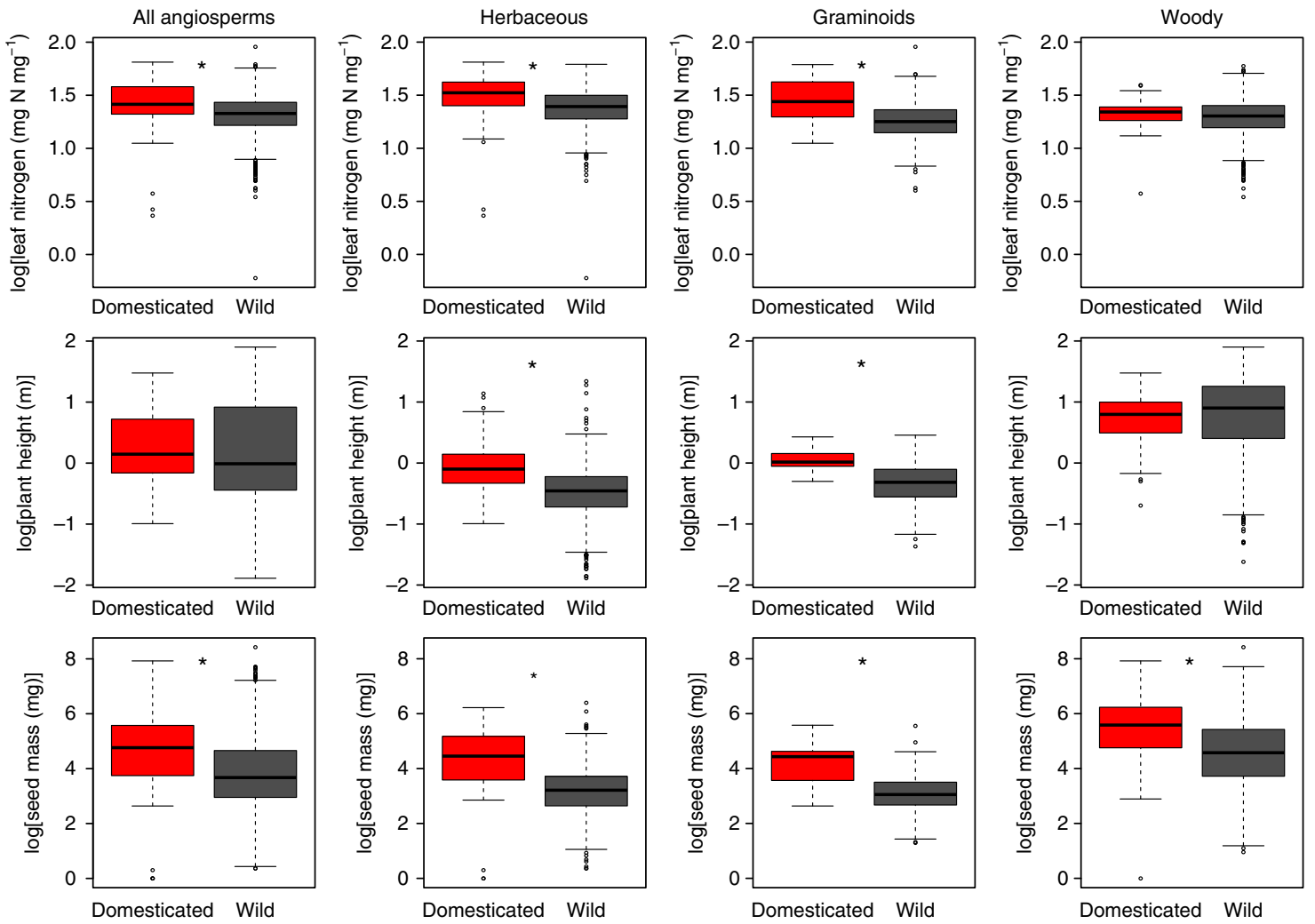

Fig. 6 | Phenotypic differentiation between crops and wild angiosperms. Separate plots are shown for all angiosperms and each growth form (that is herbaceous, graminoids and woody). Asterisks indicate statistically significant differences at $P \leq 0.05$ between domestication statuses according to phylogenetic ANOVAs (Supplementary Table 8). Centre line, median; box limits, upper and lower quartiles; whiskers, lowest/highest data point still within $1.5 \times$ interquartile range; points, data exceeding whisker bounds.

genotypic and environmental drivers, whose relative importance is plainly indistinguisible for large-scale macroecological studies. However, the few experiments that grew sets of crops and of their wild progenitors in common gardens, and phenotyped the types of traits that we measured here, tended to concur with our results, which suggests a strong genetic component at play ${ }^{22,40,47}$. Therefore, it will be necessary to take these experimental approaches one step further in terms of number of species and phylogenetic breadth, both for wild and domesticated organisms. Second, we note that, for both domesticated and wild species, intraspecific variation was not considered here. Such variation, even if unmanageable to explore systematically at the macroevolutionary scale of the current study, might expand trait spaces greatly ${ }^{48}$. Leaf-trait intraspecific variation in sunflower, wheat, maize and coffee was recently reported, and occupies a fair portion of wild phenospaces, though very rarely spreading outside wild boundaries ${ }^{40,49,50}$. We supplemented those leaf-trait patterns available in the literature with ad hoc analyses for seed and plant sizes (Supplementary Fig. 2). Similarly to leaf traits, intraspecific variation in size traits is constrained within wild envelopes in sunflower, soybean and barley (Supplementary Fig. 2). However, maize, as a crop species in which the seed-plant size centroid is outside the phenosphace of its corresponding wild analogues (Fig. 5), expands most of its intraspecific variation outside wild boundaries (Supplementary Fig. 2). In our view, these analyses, and the available literature, are still too scant to reach solid conclusions on the role of intraspecific variation in trait differences between wild and domesticated organisms. Thus, investigating how and to what extent diversifying breeding of domesticates expands phenospaces is needed to bridge the macro- and the microevolutionary scales.
This study places domesticates within their broader botanical and zoological context, which facilitates appreciation of the qualities and potentials of the species that support our food system, and could help in the search for suitable future domesticates. Suitable phenotypes among mammals include moderate-slow life histories, while fast growth traits and large size dominate among herbaceous crops. Further, the usage of a phylogenetic comparative approach, which was seldom embraced in the domestication literature (but see refs ${ }^{12,14,51}$ ), provided unique insights that are unattainable at smaller scales. Overall, our work indicates that certain phylogenetic clades and phenotypic profiles have been more exploited than others for provisioning human food, and that such filtering was based on strong, conscious or unconscious, early selection at human settlements. Future work should investigate biogeographic and historical determinants, disentangle genotypic from environmental drivers and address the microevolutionary scale of the broad phylogenetic and phenotypic patterns of differences between domesticated and wild kins revealed here.

\section{Methods}

Study system. We explored macroevolutionary patterns of the distributions of species domesticated for human food, and compared their phenotypic trait space occupancy with that of wild species. We included the broadest possible diversity of mammals and angiosperms farmed for human food provision, with distinct domestication histories and intensities, phylogenetic affinities and phenotypic profiles (see Supplementary Table 1, Supplementary Data 1, and Figs. 1-6).

Collection of data on the distribution and abundance of angiosperms and mammals farmed for food. To assess patterns of distribution and abundance of food domesticates across phylogenies, we compiled the abundances and identities of domesticated species within mammals and angiosperms, at the family and genus levels. We aimed to build a comprehensive working list of all putative species 
domesticated for food. Therefore we included all species for which farming could be substantiated, as an indicator of some degree, even if incipient, of domestication. We used the literature to build a preliminary list of mammals farmed for food ${ }^{5,6,52,53}$. We excluded most anectodal evidence of deer and antelope farming, but for the sake of inclusivity we considered species such as reindeer, sika deer, moose, bison, muskox or common eland, which are regularly farmed regionally, and thus should include incipiently domesticated populations. We also included recent incipient domesticated species for food such as the African giant rat, or the South American paca. The set of mammals comprised 27 domesticated species, distributed across 22 genera and 10 families. Taxonomy was checked using the 'taxize' R package ${ }^{54}$

For plants, we compiled a list of all putative domesticated species from the literature ${ }^{2,4,18,55}$. From that list, we extracted taxa for which cultivation could be demonstrated, and filtered that extract by species used for human food or forage. We included forage species because human food supply depends on livestock feeding, and a substantial part of the agricultural land is devoted to that usage. To assign usage for food or forage, we primarily used two studies ${ }^{2,56}$, supplemented with data from http://www.pfaf.org and other sources when needed. Plant taxonomy was standardized using the 'Taxonstand' R package ${ }^{57}$, synonymous names were cleaned and binomials were attributed to families using The Plant List v1.1 (http://www.theplantlist.org/). Thirty-five species were taxonomically unresolved, and their provisional binomials were used. Our final 944 species list should include the vast majority of angiosperms known to have been cultivated for human food. The species of our list belonged to 453 genera and 120 angiosperm families.

Domesticated mammals were grouped into two dietary types, that is, ruminants and non-ruminants, based on the MammalDIET database ${ }^{58}$, and on information at http://www.ultimateungulate.com/ungulates.html (Ruminantia). The ruminant category also included pseudo-ruminants, that is Camelidae and Hippopotamidae. Domesticated plants were grouped by growth form into herbaceous, graminoids (Poaceae, Cyperaceae and Juncaceae) and woody plants (shrubs, trees, woody vines and tree-like species). Growth forms were assigned using the TRY database ${ }^{59}$ and the Global Woodiness Database ${ }^{60}$, and were supplemented species-wise with primary literature when not available in those sources.

Selection and compilation of phenotypic traits. We selected a set of three traits for mammals and three traits for angiosperms that are functionally analogous. Selection of traits used the following criteria: (1) previous evidence of domestication effects on those traits ${ }^{14,22,29,30,36}$; (2) functional relevance for basic metabolism, resource-use, competition and reproductive strategies; and (3) availability of data, both for domesticated and for wild species. By functional analogy, the selected traits can be grouped into: (1) plant canopy height (m) and mammal adult body mass ( $\mathrm{g}$ ) as proxies for adult size and competitive ability for resources ${ }^{61,62} ;(2)$ leaf nitrogen content $\left(\mathrm{mg} \mathrm{N} \mathrm{mg}^{-1}\right)$, and size-corrected mammalian basal metabolic rate $\left(\mathrm{mlO}_{2} \mathrm{~h}^{-1} \mathrm{~g}^{-1}\right)$ as proxies of photosynthetic and metabolic rate, respectively ${ }^{26,63}$; and (3) seed dry mass $(\mathrm{mg}$ ) and neonate body mass ( $\mathrm{g}$ ) as proxies for offspring size and likelihood of survival ${ }^{64,65}$.

The two trait datasets were assembled separately for mammals and angiosperms. The dataset on mammal traits was compiled from the PanTHERIA database for adult and neonate body mass ${ }^{66}$, and from ref. ${ }^{67}$ for basal metabolic rate (supplemented with PanTHERIA if basal metabolic rate was unavailable at ref. $\left.{ }^{67}\right)$. The dataset on mammal traits comprised 480 species, including 23 domesticated species (see Supplementary Table 1 for the identity of domesticated species). For domesticated plants, trait data were not available for all 944 crops species. Thus, we started by retrieving data from the 203 species list of domesticated plants published in Meyer et al. ${ }^{18}$, which was built to maximize crop diversity. This helped to avoid biases in growth form, taxonomy or agricultural relevance. That list of crop taxa was further supplemented, again using criteria to maximize diversity and filtering by the availability of trait data. Then, data for wild angiosperms were added. Overall, the sources for angiosperm trait data were: (1) the TRY database ${ }^{59}$ (https://www.try-db.org, accessed 13 November 2016); (2) literature searches for wild species incompletely recorded or not present in the TRY database; (3) literature searches for trait data of crop species, which are mostly absent from TRY ${ }^{68}$; (4) our own data already collected on crops and other wild species (see Data availability). The final angiosperm trait dataset comprised 3,124 species, including 181 domesticated species (see Supplementary Data 1 for the identity of domesticated species).

Plant species names were standardized using the 'Taxonstand' R package ${ }^{57}$, and were attributed to families according to The Plant List v1.1 (http://www.theplantlist. org/). The majority of crop binomials are synonymous to the wild genotypes of their wild progenitors. Therefore, to decide whether a given observation of a crop-related binomial was attributable to a crop or a synonymous wild species, we used the following criteria. First, we looked for explicit statements in the original publication or database on whether the studied taxa were crop or wild. If uncertain, an observation was assigned to 'wild' if the study was observational and was conducted under natural field conditions, or if the seeds for an experiment were collected from wild stocks. In contrast, an observation was assigned to 'crop' if the seeds came from commercial companies, cultivars or varieties, or if studies had been conducted in an agricultural setting, and no explicit mention to wild status was found.
Data handling before analyses. Our angiosperm dataset had $1.51 \%$ missing data (leaf nitrogen $1.70 \%$, plant height $1.27 \%$ and seed mass $1.58 \%$ ). As several plots and analyses involved the joint use of two or more traits, we adopted a multiple imputation approach to deal with missing data, following recommended procedures ${ }^{69,70}$. We generated ten complete datasets using the predictive mean matching method of the 'mice' package ${ }^{71}$. Phylogenetic relatedness (built as described below) was incorporated into the imputation procedure as phylogenetic orthogonal eigenvectors ${ }^{72}$. Results reported in the main text are from averaged imputed data of those ten complete datasets. A dataset without imputed data, and thus with a slightly reduced sample size, was used to test for robustness and sensitivity to our data handling procedures. The results of sensitivity analyses were consistent with those shown in the main text (Supplementary Note and Supplementary Tables 12-14). The mammalian traits dataset lacked basal metabolic rate data for six species, which were estimated using the phylogenetically corrected allometric scaling of adult body mass to basal metabolic rate from ref. ${ }^{67}$ (basal metabolic rate $=2.382 \mathrm{~m}^{0.729}$ ) where $m$ is adult body mass $(\mathrm{g})$. All continuous variables were log-transformed before analyses. An exception was seed mass, which was log-generalized transformed because a few crops do not yield seeds (Musa acuminata, Vaccinium corymbosum and Allium sativum). This latter procedure is recommended when data contain zeros, and the smallest positive value is not close to one.

\section{Macroevolutionary patterns in the abundance and frequency of food}

domesticates. We performed separate analyses on the relative abundance of domesticated species, and domestication frequency, at the family and genus levels. Relative abundance, at the family and genus levels, was calculated as the number of domesticated species in a particular family or genus divided by the total number of domesticated species. Because this metric does not account for species richness within clades, we also calculated a domestication frequency metric as the number of domesticated species in a focal family or genus per total number of species in that same family or genus. These two metrics inform about different features of the distribution of domesticated species. Species richness at the family and genus levels, needed to compute domestication frequencies, was retrieved from lists of accepted names from Wilson and Reeder ${ }^{7}$ for mammals, and from The Plant List v1.1 (http://www.theplantlist.org/), making use of the 'taxonlookup' R package (https://github.com/traitecoevo/taxonlookup), for plants.

Phylogenetic hypotheses were built at the family and genus levels for mammals and for angiosperms separately. Mammal phylogenies were based on the megaphylogeny of Bininda-Emonds et al. ${ }^{73}$ as a backbone. The mammalian familylevel tree included 142 families ( $91 \%$ of total mammalian families), while the genus-level tree included 498 genera (39.6\% of total mammalian genera). Seventytwo genera were ruminants and 337 were non-ruminant herbivores (http://tolweb. org). Angiosperm phylogenies were based on the PhytoPhylo megaphylogeny ${ }^{60,74}$. The angiosperm family-level tree included 404 families (97\% of total angiosperm families), while the genus-level tree included 7,233 genera (ca. $56 \%$ of total angiosperm genera) (http://www.theplantlist.org/). All families, and all but seven genera with domesticated species, were present in the megaphylogeny. Those seven genera (Gigantochloa, Nopalea, Parmentiera, Polianthes, Sphenostylis, Stizolobium and Vitellaria) were bound to the PhytoPhylo megaphylogeny based on published phylogenies of their respective families. The genus-level tree included 2,745 genera of herbs, 421 of graminoids, 3,500 of woody plants and 567 genera hosting both herbs and woody plants. Genera containing herbs and woody plants were included in both of their respective growth-form analyses. Angiosperm phylogenies were fully resolved, but mammalian phylogenies contained some internal polytomies ( $12 \%$ of nodes in the family-level tree, $24 \%$ in ruminants and $38 \%$ in non-ruminant herbivores). Therefore, analyses were run across 100 randomly resolved familyand genus-level mammalian trees.

To assess whether the abundance and frequency of domestication are randomly distributed across mammal and angiosperm families and genera, we performed four complementary analyses. First, we conducted randomization analyses to test whether the distribution of the abundances of domesticated species per family, and of the frequencies of domestication events, differed from random expectations. Observed kurtosis and skewness of the distribution of abundances were compared with that of 1,000 randomized distributions at each respective level. Second, we computed LIPA scores based on local Moran's $I$ (ref. ${ }^{75}$ ), to detect families surrounded by phylogenetic neighbourhoods with similar or distinctive (positive or negative autocorrelation, respectively) relative abundances of domesticated species or domestication frequencies. For each LIPA score, statistical significance was assigned by performing non-parametric two-sided tests with 999 randomizations. For mammals, LIPA scores were averaged across the 100 randomly resolved trees. Third, we calculated the phylogenetic signal of the relative abundances of domesticated species, and of the frequencies of domestication, at the genus and family levels, and separately for mammals and angiosperms. Provided that our data followed either zero-inflated log-normal (abundances), or binomial (frequencies), distributions, we did not calculate standard Pagel's $\lambda$ or Blomberg's $K$ metrics, which are meant for continuous data with normal or log-normal distributions. Instead, we computed phylogenetic signal as the phylogenetic heritability parameter $(\lambda)$ of phylogenetic mixed models, where our metrics of domestication were the response, an intercept was set as the sole fixed-effects predictor, and inverse matrices of the phylogenetic distances matrices were the covariance structure terms ${ }^{76,77}$. 
Phylogenetic mixed models allow the specification of family distributions of data deviating from Gaussian. The lambda parameter of such models, specified without meaningful fixed-effect predictors, and without additional covariance structures in the random term, is the phylogenetic signal of the response variable, analogous to a null phylogenetic generalized least square mode ${ }^{76}$. Fourth, we fitted and compared two evolutionary models to test whether relative abundances and domestication frequencies were phylogenetically structured or phylogenetically independent. We used a Brownian-motion model to approximate neutral drift evolution or randomly fluctuating selection ${ }^{78}$. Under Brownian motion, relative abundances and frequencies evolve as a random walk through trait space along the branches of the phylogeny, and thus represent strong phylogenetic structuring. Brownian motion was compared with a non-phylogenetic model (a star phylogeny), which was used to approximate a phylogenetic independent distribution. To compare both models, we used the bias-corrected Akaike's information criterion $\left(\mathrm{AIC}_{\mathrm{c}}\right)$, and calculated the difference between the $\mathrm{AIC}_{\mathrm{c}}$ of the best (smallest $\mathrm{AIC}_{\mathrm{c}}$ ) and the alternative model ${ }^{79}$. In addition, for each model we calculated the $\mathrm{AIC}_{c}$ weights $\left(\mathrm{AIC}_{c}-\mathrm{w}\right)$, with a high $\mathrm{AIC}_{\mathrm{c}}-\mathrm{w}$ indicating a low relative $\mathrm{AIC}_{\mathrm{c}}$ for that model and hence higher support ${ }^{79}$. For mammals, all parameters were averaged across 100 randomly resolved trees and the percentage of preferred models was calculated. Phylogenetic signals were computed using the 'MCMCglmm' function of the R package 'MCMCglmm's , setting family distribution as zero-inflated poisson for domestication abundances, and as binomial for frequencies of domestication. Evolutionary model fitting was performed with the 'FitContinuous' function of the R package 'geiger's1. Local Moran's I was calculated using the 'lipaMoran' function of the R package 'phylosignal' ${ }^{75}$.

\section{Comparative analyses of phenotypic trait space occupancy of wild and} domesticated species. New sets of phylogenetic trees were built at the species level for those mammals and angiosperms included in our traits datasets. The mammal phylogeny for the 480 species with trait data was built from the megaphylogeny of Bininda-Emonds et al. ${ }^{73}$ using Phylomatic v.3 (ref. ${ }^{82}$ ) (http://phylodiversity.net/ phylomatic/pmws). The angiosperm phylogeny for the 3,124 species with trait data was based on the PhytoPhylo megaphylogeny ${ }^{60,74}$, and was built using scenario three of the R package 'S.PhyloMaker' ${ }^{\text {? }}$. To account for phylogenetic uncertainty (20.3\% of unresolved nodes for mammals and $15.3 \%$ for angiosperms), all analyses were performed on 100 randomly resolved trees by using the 'multi2di' function of the 'ape' R package ${ }^{83}$.

To visualize the phenotypic spaces explored by wild and domesticated species, we used bivariate phenospaces. In addition, we used convex hulls to draw the minimum convex envelope for each pair of traits, domestication status, and growth form or dietary type ${ }^{84}$. In addition, for each growth form or dietary type, we calculated the area and volume of each three-traits convex hull. To test for significant differentiation in trait space between domesticated and wild species, we performed phylogenetic-corrected multivariate analysis of variance (MANOVA) and analysis of variance (ANOVA), separately for each growth form or dietary type. Convex-hull calculations were performed using function 'convhulln' of the R package 'geometry' 85 . Phylogenetic MANOVAs and ANOVAs were run with the function 'aov.phylo' in the R package 'geiger' ${ }^{\text {'1 }}$. See Supplementary Methods for tests on whether the phenotypic departure of domesticated species from the trait medians of their wild counterparts was related to differences between domesticates in geographic origin, climate at geographic origin or antiquity of domestication. All analyses were conducted in R v3.4.3 (ref. ${ }^{86}$ ).

Reporting Summary. Further information on research design is available in the Nature Research Reporting Summary linked to this article.

\section{Data availability}

All phenotypic traits of mammalian species included in this study are available from the literature (see Methods). For plants, most data are available from the database TRY ${ }^{59}$ (https://www.try-db.org), and all original sources of TRY data are listed in Supplementary References 1. All references for data not included in TRY are available in the Supplementary References 2. Unpublished data owned by R.M. and J.M.B. are available from Supplementary Data 3. Unpublished data from the University of Sheffield database of weed functional attributes can be requested from G.J. Lists of livestock and crop taxa are available in Supplementary Table 1 and Supplementary Data 1, respectively. Phylogenetic trees used in this study are available in Supplementary Data 4. Data on geography and climate at domestication sites are available as Supplementary Data 5.

Received: 9 July 2017; Accepted: 6 September 2018; Published online: 22 October 2018

\section{References}

1. RBG Kew. The State of the World's Plants Report - 2016 (Royal Botanic Gardens, Kew, London, 2016).

2. Zeven, A. C. \& De Wet, J. M. J. Dictionary of Cultivated Plants and Their Regions of Diversity: Excluding Most Ornamentals, Forest Trees and Lower Plants (Pudoc, Wageningen, 1982); http://agris.fao.org/agris-search/search. do? recordID $=$ XE8282385
3. Dirzo, R. \& Raven, P. H. Global state of biodiversity and loss. Annu. Rev. Environ. Resour. 28, 137-167 (2003).

4. Meyer, R. S. \& Purugganan, M. D. Evolution of crop species: genetics of domestication and diversification. Nat. Rev. Genet. 14, 840-852 (2013).

5. Zeder, M. A. in Biodiversity in Agriculture: Domestication, Evolution, and Sustainability (eds Gepts, P. et al.) 227-259 (Cambridge Univ. Press, New York, 2012).

6. Clutton-Brock, J. A Natural History of Domesticated Mammals (Cambridge Univ. Press, New York, 1999).

7. Wilson, D. \& Reeder, D. A. Mammal Species of the World (Johns Hopkins Univ. Press, Baltimore, 2005).

8. Gepts, P. in Plant Breeding Reviews Vol. 24 (ed. Janick, J.) Ch. 1, 1-44 (John Wiley \& Sons, Hoboken, 2010).

9. Blomberg, S. P. \& Garland, T. Jr. Tempo and mode in evolution: phylogenetic inertia, adaptation and comparative methods. J. Evol. Biol. 15, 899-910 (2002).

10. Diamond, J. Evolution, consequences and future of plant and animal domestication. Nature 418, 700-707 (2002).

11. Salman-Minkov, A., Sabath, N. \& Mayrose, I. Whole-genome duplication as a key factor in crop domestication. Nat. Plants 2, 16115 (2016).

12. Whitehead, S. R., Turcotte, M. M. \& Poveda, K. Domestication impacts on plant-herbivore interactions: a meta-analysis. Phil. Trans. R. Soc. B 372, 20160034 (2017).

13. Milla, R., Osborne, C. P., Turcotte, M. M. \& Violle, C. Plant domestication through an ecological lens. Trends Ecol. Evol. 30, 463-469 (2015).

14. Preece, C. et al. Were Fertile Crescent crop progenitors higher yielding than other wild species that were never domesticated? New Phytol. 207, 905-913 (2015).

15. Darwin, C. The Variation of Animals and Plants under Domestication Vol. 2 (O. Judd, London, 1868)

16. Abbo, S. et al. Plant domestication versus crop evolution: a conceptual framework for cereals and grain legumes. Trends Plant Sci. 19, 351-360 (2014)

17. Harlan, J. R. Crops and Man (American Society of Agronomy, Madison, 1992).

18. Meyer, R. S., DuVal, A. E. \& Jensen, H. R. Patterns and processes in crop domestication: an historical review and quantitative analysis of 203 global food crops. New Phytol. 196, 29-48 (2012).

19. Sánchez-Villagra, M. R., Geiger, M. \& Schneider, R. A. The taming of the neural crest: a developmental perspective on the origins of morphological covariation in domesticated mammals. R. Soc. Open Sci. 3, 160107 (2016).

20. Larson, G. \& Fuller, D. Q. The evolution of animal domestication. Annu. Rev. Ecol. Evol. Syst. 45, 115-136 (2014).

21. Reich, P. B. The world-wide 'fast-slow'plant economics spectrum: a traits manifesto. J. Ecol. 102, 275-301 (2014).

22. Milla, R., Morente-López, J., Alonso-Rodrigo, J. M., Martín-Robles, N. \& Chapin, F. S. III. Shifts and disruptions in resource-use trait syndromes during the evolution of herbaceous crops. Proc. R. Soc. B 281, 20141429 (2014)

23. Tribouillois, H. et al. A functional characterisation of a wide range of cover crop species: growth and nitrogen acquisition rates, leaf traits and ecological strategies. PLoS ONE 10, e0122156 (2015)

24. Milla, R. \& Matesanz, S. Growing larger with domestication: a matter of physiology, morphology or allocation? Plant Biol. 19, 475-483 (2017)

25. Díaz, S. et al. The global spectrum of plant form and function. Nature 529, 167-171 (2015).

26. Ricklefs, R. E. \& Wikelski, M. The physiology/life-history nexus. Trends Ecol. Evol. 17, 462-468 (2002).

27. Lovegrove, B. G. The zoogeography of mammalian basal metabolic rate. Am. Nat. 156, 201-219 (2000).

28. Lovegrove, B. G. The influence of climate on the basal metabolic rate of small mammals: a slow-fast metabolic continuum. J. Comp. Physiol. B 173 , $87-112(2003)$

29. Zohary, D., Tchernov, E. \& Horwitz, L. K. The role of unconscious selection in the domestication of sheep and goats. J. Zool. 245, 129-135 (1998).

30. Cunniff, J. et al. Functional traits differ between cereal crop progenitors and other wild grasses gathered in the Neolithic Fertile Crescent. PLOS ONE 9, e87586 (2014).

31. Biro, P. \& Stamps, J. Are animal personality traits linked to life-history productivity? Trends Ecol. Evol. 23, 361-368 (2008).

32. Dittmann, M. T. et al. Characterising an artiodactyl family inhabiting arid habitats by its metabolism: low metabolism and maintenance requirements in camelids. J. Arid Environ. 107, 41-48 (2014).

33. Careau, V., Bininda-Emonds, O. R. P., Thomas, D. W., Réale, D. \& Humphries, M. M. Exploration strategies map along fast-slow metabolic and life-history continua in muroid rodents. Funct. Ecol. 23 150-156 (2009).

34. Réale, D. et al. Personality and the emergence of the pace-of-life syndrome concept at the population level. Phil. Trans. R. Soc. B 365 , 4051-4063 (2010) 
35. Found, R. \& St. Clair, C. C. Ambidextrous ungulates have more flexible behaviour, bolder personalities and migrate less. Open Sci. 4, 160958 (2017)

36. Tchernov, E. \& Horwitz, L. K. Body size diminution under domestication: unconscious selection in primeval domesticates. J. Anthropol. Archaeol. 10, 54-75 (1991).

37. Rensch, B. Evolution Above the Species Level (Columbia Univ. Press, New York, 1960)

38. Vigne, J.-D. The origins of animal domestication and husbandry: a major change in the history of humanity and the biosphere. C. R. Biol. 334, 171-181 (2011).

39. Li, Y. et al. Habitat filtering determines the functional niche occupancy of plant communities worldwide. J. Ecol. 106, 1001-1009 (2018).

40. Donovan, L. A., Mason, C. M., Bowsher, A. W., Goolsby, E. W. \& Ishibashi, C. D. A. Ecological and evolutionary lability of plant traits affecting carbon and nutrient cycling. J. Ecol. 102, 302-314 (2014).

41. Rotundo, J. L. \& Cipriotti, P. A. Biological limits on nitrogen use for plant photosynthesis: a quantitative revision comparing cultivated and wild species. New Phytol. 214, 120-131 (2017).

42. Denison, R. F. Darwinian Agriculture: How Understanding Evolution Can Improve Agriculture (Princeton Univ. Press, Princeton, 2012).

43. Miller, A. J. \& Gross, B. L. From forest to field: perennial fruit crop domestication. Am. J. Bot. 98, 1389-1414 (2011).

44. Brown, J. \& West, G. Scaling in Biology (Oxford Univ. Press, Oxford, 2000).

45. West, G. B., Brown, J. H. \& Enquist, B. J. General model for the origin of allometric scaling laws in biology. Science 276, 122-126 (1997).

46. Reich, P., Tjoelker, M., Machado, J.-L. \& Oleksyn, J. Universal scaling of respiratory metabolism, size and nitrogen in plants. Nature 439, 457-461 (2006).

47. Preece, C. et al. How did the domestication of Fertile Crescent grain crops increase their yields? Funct. Ecol. 31, 387-397 (2017)

48. Vasseur, F., Violle, C., Enquist, B. J., Granier, C. \& Vile, D. A common genetic basis to the origin of the leaf economics spectrum and metabolic scaling allometry. Ecol. Lett. 15, 1149-1157 (2012).

49. Martin, A. R. et al. Inter- and intraspecific variation in leaf economic traits in wheat and maize. AoB Plants 10, ply006 (2018).

50. Martin, A. et al. Intraspecific trait variation across multiple scales: the leaf economics spectrum in coffee. Funct. Ecol. 31, 604-612 (2017).

51. Diamond, J. Guns, Germs, and Steel: The Fates of Human Societies (W. W. Norton, London, 1997)

52. Smythe, N. \& Brown de Guanti, O. Domestication and Husbandry of the Paca (Agouti paca) FAO conservation guide 26 (Food and Agriculture Organization of the United Nations, Rome, 1995).

53. Moreira, J. R. \& Pinheiro, M. S. in Capybara: Biology, Use and Conservation of an Exceptional Neotropical Species (eds Moreira, J. R. et al.) 333-344 (Springer, New York, 2013).

54. Chamberlain, S. A. \& Szocs, E. taxize: taxonomic search and retrieval in R. F1000Research 2, 191 (2013).

55 . Vincent, $H$. et al. A prioritized crop wild relative inventory to help underpin global food security. Biol. Conserv. 167, 265-275 (2013).

56. Mansfeld, R. \& Hanelt, P. Mansfeld's Encyclopedia of Agricultural and Horticultural Crops (Springer, Berlin, 2001).

57. Cayuela, L., Granzow-de la Cerda, Í., Albuquerque, F. S. \& Golicher, D. J. Taxonstand: an $\mathrm{R}$ package for species names standardisation in vegetation databases. Methods Ecol. Evol. 3, 1078-1083 (2012).

58. Kissling, W. D. et al. Establishing macroecological trait datasets: digitalization, extrapolation, and validation of diet preferences in terrestrial mammals worldwide. Ecol. Evol. 4, 2913-2930 (2014).

59. Kattge, J. et al. TRY - a global database of plant traits. Global Change Biol. 17, 2905-2935 (2011).

60. Zanne, A. E. et al. Three keys to the radiation of angiosperms into freezing environments. Nature 506, 89-92 (2014).

61. Moles, A. T. et al. Global patterns in plant height. J. Ecol. 97, 923-932 (2009)

62. Glazier, D. S. \& Eckert, S. E. Competitive ability, body size and geographical range size in small mammals. J. Biogeogr. 29, 81-92 (2002).

63. Wright, I. J. et al. The worldwide leaf economics spectrum. Nature $\mathbf{4 2 8}$ 821-827 (2004).

64. Moles, A. T. et al. A brief history of seed size. Science 307, 576-580 (2005).

65. Riesch, R., Martin, R. A., Lerp, H., Plath, M. \& Wronski, T. Size and sex matter: reproductive biology and determinants of offspring survival in Gazella marica. Biol. J. Linn. Soc. 110, 116-127 (2013).

66. Jones, K. E. et al. PanTHERIA: a species-level database of life history, ecology, and geography of extant and recently extinct mammals. Ecology 90, 2648 (2009).

67. Genoud, M., Isler, K. \& Martin, R. Comparative analyses of basal rate of metabolism in mammals: data selection does matter. Biol. Rev. 93, 404-438 (2018).

68. Martin, A. R. \& Isaac, M. E. Plant functional traits in agroecosystems: a blueprint for research. J. Appl. Ecol. 52, 1425-1435 (2015).

69. Nakagawa, S. \& Freckleton, R. P. Missing inaction: the dangers of ignoring missing data. Trends Ecol. Evol. 23, 592-596 (2008)
70. Penone, C. et al. Imputation of missing data in life-history trait datasets: which approach performs the best? Methods Ecol. Evol. 5, 961-970 (2014).

71. van Buuren, S. \& Groothuis-Oudshoorn, K. mice: multivariate imputation by chained equations in R. J. Stat. Softw. 45, 1-67 (2011).

72. Santos, T. PVR: phylogenetic eigenvectors regression and phylogentic signal-representation curve. R package version 0.3 (2018); https:// cran.r-project.org/web/packages/PVR/index.html

73. Bininda-Emonds, O. R. P. et al. The delayed rise of present-day mammals. Nature 446, 507-512 (2007)

74. Qian, H. \& Jin, Y. An updated megaphylogeny of plants, a tool for generating plant phylogenies and an analysis of phylogenetic community structure. J. Plant Ecol. 9, 233-239 (2016).

75. Keck, F., Rimet, F., Bouchez, A. \& Franc, A. phylosignal: an R package to measure, test, and explore the phylogenetic signal. Ecol. Evol. 6, 2774-2780 (2016).

76. de Villemereuil, P. \& Nakagawa, S. in Modern Phylogenetic Comparative Methods and Their Application in Evolutionary Biology (ed. Garamszegi, L.) 287-304 (Springer-Verlag, Berlin, 2014).

77. Hadfield, J. \& Nakagawa, S. General quantitative genetic methods for comparative biology: phylogenies, taxonomies and multi-trait models for continuous and categorical characters. J. Evol. Biol. 23 , 494-508 (2010).

78. Felsenstein, J. Phylogenies and quantitative characters. Annu. Rev. Ecol. Syst. 19, 445-471 (1988).

79. Burnham, K. P. \& Anderson, D. R. Model Selection and Multimodel Inference: A Practical Information-theoretic Approach (Springer-Verlag, New York, 2002).

80. Hadfield, J. MCMC methods for multi-response generalised linear mixed models: the MCMCglmm R package. J. Stat. Softw. 33, $1-22$ (2010).

81. Harmon, L. J., Weir, J. T., Brock, C. D., Glor, R. E. \& Challenger, W. GEIGER: investigating evolutionary radiations. Bioinformatics 24, 129-131 (2007)

82. Webb, C. O. \& Donoghue, M. J. Phylomatic: tree assembly for applied phylogenetics. Mol. Ecol. Resour. 5, 181-183 (2005).

83. Paradis, E., Claude, J. \& Strimmer, K. APE: analyses of phylogenetics and evolution in R language. Bioinformatics 20, 289-290 (2004).

84. Cornwell, W. K., Schwilk, D. W. \& Ackerly, D. D. A trait-based test for habitat filtering: convex hull volume. Ecology 87, 1465-1471 (2006).

85. Barber, C. B. et al. geometry: mesh generation and surface tessellation. R package version 0.3-4 (2014); https://cran.r-project.org/web/packages/ geometry/index.html

86. R Core Team. R: a language and environment for statistical computing (R Foundation for Statistical Computing, 2016); https://www.r-project.org/

\section{Acknowledgements}

R.M., J.C.-L. and J.M.B. were funded by grants CGL2014-56567-R, CGL2017-83855-R and PCIN-2014-053 (Ministerio de Economia y Competitividad, MINECO, Spain), and Eco-serve project (Biodiversa-FACCE, Horizon 2020, European Union). The study has been supported by the TRY initiative on plant traits (http://www.try-db.org). The TRY initiative and database is hosted, developed and maintained by J. Kattge and G. Bönisch (Max Planck Institute for Biogeochemistry, Jena, Germany). TRY is currently supported by DIVERSITAS/Future Earth and the German Centre for Integrative Biodiversity Research (iDiv) Halle-Jena -Leipzig. R.M. thanks C. F. Ingala from Universidad Rey Juan Carlos. E.E.S. thanks the FAPESP/BIOTA programme for financial support. J.K. thanks BACI (EU grant ID 640176). T.H. thanks the support of Australian Research Council (DP130013029). V.D.P. was supported by CNPq, Brazil, grant no. 307689/2014-0, K.K. was funded by the project Resilient Forests (KB-29-009-003).

\section{Author contributions}

R.M. and J.M.B. designed the study and compiled the data. R.M., J.M.B., J.C.-L. and M.M.T. performed statistical analyses. R.M. and J.M.B. wrote a first draft of the paper. M.M.T., G.J., C.P.O. and C.V. extensively revised drafts. All authors contributed to the writing of, and approved, the final version.

\section{Competing interests}

The authors declare no competing interests.

\section{Additional information}

Supplementary information is available for this paper at https://doi.org/10.1038/ s41559-018-0690-4.

Reprints and permissions information is available at www.nature.com/reprints. Correspondence and requests for materials should be addressed to R.M.

Publisher's note: Springer Nature remains neutral with regard to jurisdictional claims in published maps and institutional affiliations.

(c) The Author(s), under exclusive licence to Springer Nature Limited 2018 


\section{Reporting Summary}

Nature Research wishes to improve the reproducibility of the work that we publish. This form provides structure for consistency and transparency in reporting. For further information on Nature Research policies, see Authors \& Referees and the Editorial Policy Checklist.

\section{Statistical parameters}

When statistical analyses are reported, confirm that the following items are present in the relevant location (e.g. figure legend, table legend, main text, or Methods section).

$\mathrm{n} / \mathrm{a} \mid$ Confirmed

$\square$ The exact sample size $(n)$ for each experimental group/condition, given as a discrete number and unit of measurement

$\square$ \ An indication of whether measurements were taken from distinct samples or whether the same sample was measured repeatedly

$\square$ The statistical test(s) used AND whether they are one- or two-sided

Only common tests should be described solely by name; describe more complex techniques in the Methods section.

$\square$ A description of all covariates tested

$\square$ \A description of any assumptions or corrections, such as tests of normality and adjustment for multiple comparisons

$\square$ A full description of the statistics including central tendency (e.g. means) or other basic estimates (e.g. regression coefficient) AND

$\triangle$ variation (e.g. standard deviation) or associated estimates of uncertainty (e.g. confidence intervals)

$\square$ For null hypothesis testing, the test statistic (e.g. $F, t, r$ ) with confidence intervals, effect sizes, degrees of freedom and $P$ value noted

Give P values as exact values whenever suitable.

Х $\square$ For Bayesian analysis, information on the choice of priors and Markov chain Monte Carlo settings

\ $\square$ For hierarchical and complex designs, identification of the appropriate level for tests and full reporting of outcomes

$\bigotimes \square$ Estimates of effect sizes (e.g. Cohen's $d$, Pearson's $r$ ), indicating how they were calculated

$\varnothing$ Clearly defined error bars

State explicitly what error bars represent (e.g. SD, SE, Cl)

Our web collection on statistics for biologists may be useful.

\section{Software and code}

Policy information about availability of computer code

Data collection code for retrieving data was only used to gather data on geographical coordinates and climate at origin of wild progenitors (sources for code described in Supplementary Methods)

Data analysis

sources of R code used for all data analyses of this work are described and referenced in the manuscript (Methods and Suppl.Methods sections)

For manuscripts utilizing custom algorithms or software that are central to the research but not yet described in published literature, software must be made available to editors/reviewers upon request. We strongly encourage code deposition in a community repository (e.g. GitHub). See the Nature Research guidelines for submitting code \& software for further information.

\section{Data}

Policy information about availability of data

All manuscripts must include a data availability statement. This statement should provide the following information, where applicable:

- Accession codes, unique identifiers, or web links for publicly available datasets

- A list of figures that have associated raw data

- A description of any restrictions on data availability

All phenotypic traits of mammalian species included in this study are available from the literature (see Methods section). For plants, most data are available from 
the database TRY (https://www.try-db.org), and all original sources of TRY data are listed in Supplementary References 1. All references for data not included in TRY are available in the Supplementary References 2. Unpublished data owned by R.M. and J.M.B. are available from Supplementary Data 3. Unpublished data from the University of Sheffield database of weed functional attributes can be requested from G.J. Lists of livestock and crop taxa are available at Supplementary Table 1 and Supplementary Data 1, respectively. Phylogenetic trees used in this study are available from Supplementary Data 4 . Data on geography and climate at domestication sites are available as Supplementary Data 5.

\section{Field-specific reporting}

Please select the best fit for your research. If you are not sure, read the appropriate sections before making your selection.

Life sciences

Behavioural \& social sciences

Ecological, evolutionary \& environmental sciences

For a reference copy of the document with all sections, see nature.com/authors/policies/ReportingSummary-flat.pdf

\section{Ecological, evolutionary \& environmental sciences study design}

All studies must disclose on these points even when the disclosure is negative.

Study description

Sampling strategy

Data collection

Timing and spatial scale

Data exclusions

Reproducibility

Randomization

Blinding
We explored macroevolutionary patterns of the distributions of species domesticated for human food, and compared their phenotypic trait space occupancy with that of wild species. We included the broadest possible diversity of mammals and angiosperms farmed for human food provision, with distinct domestication histories and intensities, phylogenetic affinities, and phenotypic profiles (see Supplementary Table 1, Supplementary Data 1, and Figures 1-6 of the ms).

species of crops and livestock mammals at the global scale, plus 3,124 wild angiosperms and 480 sps of wild mammals

This project is a macroevolutionary analysis, thus the number of species in sub-groups (domesticates/wilds, different plant growth forms or animal dietary guilds) was set by group size in nature, and data availabilty. No sample-size calculation was thus performed. We collected a large proportion of all domesticated animals and cultivated plants useful for human subsistence. Those sets of domesticated species were used to explore the distribution of relative abundances of domesticated species and domestication frequencies across their respective phylogenies. Further, In order to explore phenotypic spaces and phenotypic differentiation, we built two new datasets for each group (i.e. mammals and angiosperms) following diversity criteria (which helped to avoid any biases in growth form, taxonomic, agronomic or productivity relevance) and focal traits availability. In this second set of analises, the mammalian dataset contained 23 livestock species and 480 wild mammals, while the angiosperms dataset involved 181 crops and 3,124 wild plants.

this was a compilatory project. In the body of the ms. we disclose in full detail how we compiled the data and data sources. Authors responsible for data compilation are R.M and J.M.B.

global

No data were excluded from the analyses.

Becasue of topological phylogenetic uncertainty some analyses were ran over 100 randomly-resolved phylogenies. Thus, phylogenetic signals and Local Moran's I for relative abundance and domestication frequency in the mammals dataset were performed over 100 randomly-resolved phylogenies. Phylogenetic MANOVAs and ANOVAs for mammals and angiosperms dataset also were performed on 100 randomly-resolved phylogenies. In both cases the percentaje of significant tests for each comparison are indicated.

Species observations were not randomly allocated into "wild" or "domesticated" states. This is not relevant for this study since an observation was ascribed to "wild" or "domesticated" based on predefined criteria.

n/a 
Materials \& experimental systems

$\mathrm{n} / \mathrm{a}$ Involved in the study

\ $\square$ Unique biological materials

Х $\square$ Antibodies

\ $\square$ Eukaryotic cell lines

Х $\square$ Palaeontology

$\triangle \square$ Animals and other organisms

$\bigotimes \mid \square$ Human research participants
Methods

$\mathrm{n} / \mathrm{a}$ Involved in the study

$\triangle(\square$ ChIP-seq

Х $\square$ Flow cytometry

$\searrow \mid \square$ MRI-based neuroimaging 